\title{
Optimization of the Gain-Bandwidth Product of Capacitive Micromachined Ultrasonic Transducers
}

\author{
Selim Olcum, Student Member, IEEE, Muhammed N. Senlik, Student Member, IEEE, \\ and Abdullah Atalar, Senior Member, IEEE
}

\begin{abstract}
Capacitive micromachined ultrasonic transducers (cMUT) have large bandwidths, but they typically have low conversion efficiencies. This paper defines a performance measure in the form of a gain-bandwidth product and investigates the conditions in which this performance measure is maximized. A Mason model corrected with finite-element simulations is used for the purpose of optimizing parameters. There are different performance measures for transducers operating in transmit, receive, or pulse-echo modes. Basic parameters of the transducer are optimized for those operating modes. Optimized values for a cMUT with silicon nitride membrane and immersed in water are given. The effect of including an electrical matching network is considered. In particular, the effect of a shunt inductor in the gain-bandwidth product is investigated. Design tools are introduced, which are used to determine optimal dimensions of cMUTs with the specified frequency or gain response.
\end{abstract}

\section{INTRODUCTION}

$\mathrm{C}$ APACITIVE micromachined ultrasonic transducers (cMUTs) [1]-[3] have the potential of replacing piezoelectric transducers in many areas. The applications include air-coupled nondestructive testing [4], [5], medical imaging [6], [7], three-dimensional (3-D) immersion imaging with 2-D transducer arrays [8], flow meters, level meters, position and distance measurements and microphones. Recently, analytical and computational models for the cMUTs have been developed [9]-[12]. Drawbacks of the cMUTs are studied and eliminated for optimum performance for a variety of applications. Increasing the dynamic range, decreasing parasitic capacitances and crosscoupling [13] have been the major goals. The methods to overcome the problems include new ways of electrode patterning [14], [15], changing the material used for membrane, optimizing the geometry for the best performance, and finding new regimes of operation [16].

It is shown that a large bandwidth is possible with an untuned cMUT immersed in water [10], [14]. For such a cMUT, the operation frequency range may extend from very low frequencies to the antiresonance of the membrane [17]. However, those cMUTs have small conversion efficiencies and are not as sensitive as piezoelectric transducers.

Manuscript received August 3, 2004; accepted January 4, 2004.

The authors are with the Electrical and Electronics Engineering Department, Bilkent University, Ankara Turkey (e-mail: selim@ee.bilkent.edu.tr).
An electrical tuning network can be added to increase the gain. In this work, we explore the limits of a cMUT operating in different regimes using the Mason model corrected with finite-element method (FEM) simulations. We try to maximize the bandwidth of a cMUT while keeping the output pressure or the conversion efficiency at a reasonable value. For this purpose, we define performance measures in the form of a pressure-bandwidth product or a gain-bandwidth product. We try to maximize this figure of merit by optimizing various geometrical parameters of the cMUT.

\section{Mason Model Corrected By Finite-Element Method Simulations}

A cross-sectional view of a cMUT is seen in Fig. 1, in which $t_{m}$ is the thickness of the membrane, $t_{i}$ is the thickness of the insulator on the bulk silicon. The radius of the membrane is represented by $a$ and the gap height is symbolized by $t_{g}$. An electrode is placed on the bottom of the membrane. The cMUTs are assumed to be fabricated from silicon nitride.

It is customary to use a Mason model for the cMUTs as depicted in Fig. 2 [18]. In this model, the left-hand side of the transformer is electrical, and the right-hand side is mechanical. The mechanical impedance of the membrane, $Z_{m}$, is approximated by the ratio of the applied pressure on the membrane to the average velocity of the membrane. Because the average velocity of the membrane is a function of the excitation frequency, $Z_{m}$ is a function of frequency. The sign errors in the relation given in [10] are corrected to give the membrane impedance as:

$$
Z_{m}=\frac{j w \rho t_{m} a k_{1} k_{2}\left(k_{1} J_{10}-k_{2} J_{01}\right)}{a k_{1} k_{2}\left(k_{1} J_{10}-k_{2} J_{01}\right)-2\left(k_{1}^{2}+k_{2}^{2}\right) J_{11}}
$$

where $J_{01}=J_{0}\left(k_{1} a\right) J_{1}\left(k_{2} a\right), J_{10}=J_{1}\left(k_{1} a\right) J_{0}\left(k_{2} a\right), J_{11}=$ $J_{1}\left(k_{1} a\right) J_{1}\left(k_{2} a\right), \omega$ is the radian frequency, $\rho$ is the density of the membrane material, $J_{0}$ and $J_{1}$ are the zeroth and first-order Bessel functions of the first kind. $k_{1}$ and $k_{2}$ are given by:

$$
k_{1}=\sqrt{\frac{\sqrt{d^{2}+4 c \omega^{2}}-d}{2 c}} \text { and } k_{2}=j \sqrt{\frac{\sqrt{d^{2}+4 c \omega^{2}}+d}{2 c}},
$$




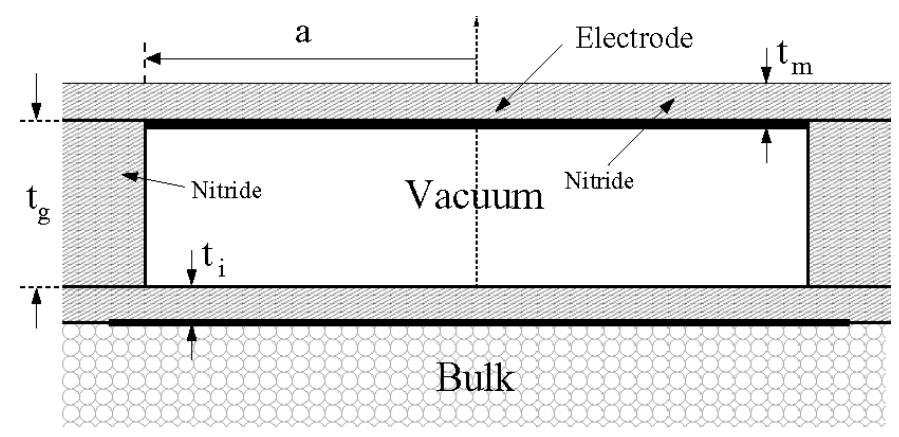

Fig. 1. Cross-sectional view of a cMUT.

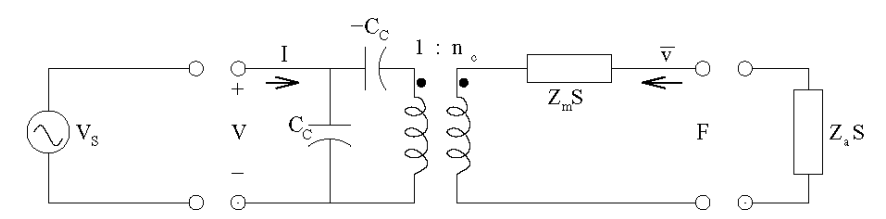

(a) Transmitter cMUT
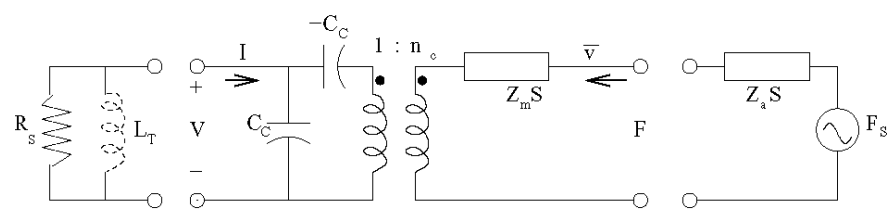

(b) Receiver cMUT

Fig. 2. Mason model (a) for a cMUT operating as a transmitter excited by a voltage source $\left(V_{S}\right)$ to drive the acoustic impedance of the immersion medium $\left(Z_{a} S\right)$ (b) for a cMUT operating as a receiver excited by the acoustical source $\left(F_{S}, Z_{a} S\right)$ to drive the electrical load resistance of the receiver circuitry $\left(R_{S}\right) . S$ is the area of the transducer, $L_{T}$ is the tuning inductor.

where

$$
c=\frac{\left(Y_{0}+T\right) t_{m}^{2}}{12 \rho\left(1-\sigma^{2}\right)} \text { and } d=\frac{T}{\rho},
$$

here, $Y_{0}$ is the Young's modulus, $T$ is the residual stress, and $\sigma$ is the Poisson's ratio of the membrane material. Around its first natural resonance frequency, $Z_{m}$ can be modeled by a mass and a spring system. The effective mass, $m_{e}$, of the membrane can be related to the actual mass of the membrane using the slope of $Z_{m}$ around the resonance as:

$$
m_{e} \simeq 1.8 \rho t_{m} \pi a^{2} .
$$

Another electrical parameter that depends on the physical dimensions is the shunt input capacitance at the electrical port. The value of this capacitance can be found by the parallel plate approximation [19]:

$$
C_{c} \simeq 2 \pi \epsilon_{0} \int_{0}^{a+\bar{t}_{g}} \frac{r}{\bar{t}_{g}-x(r)} d r
$$

where $\bar{t}_{g}$ is the effective gap height $\bar{t}_{g}=t_{g}+\epsilon_{0} t_{i} / \epsilon . \epsilon_{0}$ and $\epsilon$ are the permittivity constants of air and insulator material, respectively. $x(r)$ is the deflection profile of the membrane as determined by FEM simulations. The extra capacitance due to fringing fields is included approximately by extending the radius from $a$ to $a+\bar{t}_{g}$. The accuracy of the model is tested for the gap height values between $0.1 \mu \mathrm{m}$ and $1 \mu \mathrm{m}$. The resulting capacitance values are within $1 \%$ of the corresponding FEM simulations. Note that the membrane thickness, $t_{m}$, does not affect the value of $C_{c}$, as the electrode is placed under the membrane.

Typically, many cMUT cells are connected together to form a transducer. An extra capacitance arises because of the interconnections between the cMUT electrodes. Although this spurious capacitor can be quite large, in this work we will ignore it for simplicity. Therefore, our results are somewhat optimistic.

Because the turns ratio, $n_{c}$, is the product of the capacitance with the electric field [17], $n_{c}$ for a deflected membrane can be determined by the following integration:

$$
n_{c} \simeq 2 \pi \epsilon_{0} V_{D C} \int_{0}^{a+\bar{t}_{g}} \frac{r}{\left(\bar{t}_{g}-x(r)\right)^{2}} d r
$$

where $V_{D C}$ is the applied bias voltage.

The first natural resonance frequency can be written in terms of the effective mass, $m_{e}$, and the stiffness, $\kappa$, [14] of the membrane as:

$$
f_{r}=\frac{1}{2 \pi} \sqrt{\frac{\kappa}{m_{e}}} \simeq \frac{2 t_{m}}{\pi a^{2}} \sqrt{\frac{Y_{0}+T}{1.8 \rho\left(1-\sigma^{2}\right)}} .
$$

An approximate expression for the collapse voltage is:

$$
V_{\text {col }} \simeq 0.7 \sqrt{\frac{128\left(Y_{0}+T\right) t_{m}^{3} \bar{t}_{g}^{3}}{27 \epsilon_{0}\left(1-\sigma^{2}\right) a^{4}}}
$$

A more accurate value for $V_{\text {col }}$ can be determined using the method developed in [12]. A fast numerical algorithm is implemented using the parallel plate force distribution. We determined $V_{c o l}$ and the deflection profile, $x(r)$, of the membrane for direct current (DC) biased operation. The resulting $V_{c o l}$ values are within $5 \%$ of the results obtained with FEM simulations ${ }^{1}$.

We checked the validity of the model by comparing with the experimental results of [20]. They measured $f_{r}=$ $12 \mathrm{MHz}$ with a $12 \mathrm{MHz}$ bandwidth. Our predictions for the same geometry ${ }^{2}$ and with material constants given in Table I are as follows: $f_{r}=13.1 \mathrm{MHz}$, bandwidth $=13.7 \mathrm{MHz}$ (2.3 MHz to $16 \mathrm{MHz}$ ), one-way conversion loss $=12.2 \mathrm{~dB}$.

It was shown [21] that the effect of liquid loading in a liquid immersed cMUT is not negligible, especially if the membrane is thin. With liquid loading, the resonance frequency shifts to lower frequencies. Nevertheless, we ignored this effect for the sake of simplicity.

${ }^{1}$ FEM simulations are done with ANSYS (ANSYS Inc., Canonsburg, PA). Two electrostatic analyses are followed by two prestressed harmonic analyses [14] calculating the mechanical impedance and the turns ratio of a cMUT.

${ }^{2} a=18 \mu \mathrm{m}, t_{m}=0.88 \mu \mathrm{m}, t_{g}=0.12 \mu \mathrm{m}, t_{i}=0.2 \mu \mathrm{m}, T=0$, $R_{S}=220 \mathrm{k} \Omega$. 
TABLE I

Constant Parameters Used in the Simulations.

\begin{tabular}{ll}
\hline Parameter & Value \\
\hline Young's modulus of $\mathrm{Si}_{3} \mathrm{~N}_{4}, Y_{0}$ & $3.2 \times 10^{5} \mathrm{MPa}$ \\
Poisson's ratio of $\mathrm{Si}_{3} \mathrm{~N}_{4}, \sigma$ & 0.263 \\
Relative permittivity of $\mathrm{Si}_{3} \mathrm{~N}_{4}, \epsilon_{r n}$ & 5.7 \\
Density of $\mathrm{Si}_{3} \mathrm{~N}_{4}, \rho$ & $3.27 \mathrm{~g} / \mathrm{cm}^{3}$ \\
Breakdown voltage of $\mathrm{Si}_{3} \mathrm{~N}_{4}$ & $900 \mathrm{~V} / \mu \mathrm{m}$ \\
\hline
\end{tabular}

\section{Optimization of Performance}

If the membrane of a cMUT is very thin, the mechanical impedance, $Z_{m}$, of the membrane is very low compared to the acoustical impedance of the immersion medium, $Z_{a}$; hence, $Z_{m}$ can be ignored. In this case, the Mason model reduces to just a resistance-capacitance (RC) circuit, in which bandwidth can be made very large at the expense of gain. In this work, we do not ignore $Z_{m}$. We will explore the effect of various device dimensions on the overall circuit. In particular, we would like to optimize the radius $(a)$, the thickness of the membrane $\left(t_{m}\right)$, the gap height $\left(t_{g}\right)$, and the electrical termination resistance $\left(R_{S}\right) . Z_{m}$ and $n_{c}$ are dependent on the above parameters as discussed in Section II. The mechanical termination impedance, $Z_{a} S$, is dependent on $Z_{a}$ as well as the area of the membrane, $S$. To make a fair comparison of cMUTs with different dimensions, we always choose the maximum applied voltage as $0.9 V_{c o l}$ of the corresponding membrane.

The electrical side termination impedances of cMUTs for transmission and receive modes can be different. Typically, a low resistance electrical source is used in the transmission mode. In the receive mode, the optimal electrical termination impedance may be relatively high $(10 \mathrm{~K} \Omega$ to $100 \mathrm{~K} \Omega$ per unit cMUT). Therefore, transmission and receive modes must be treated separately, although cMUT is a reciprocal device.

\section{A. Transmission Mode}

A cMUT used in transmission mode has a limitation in the applied voltage due to breakdown of insulation material or the collapse voltage of the membrane. Other than this limit, there is no practical limitation in the amount of available electrical power. Moreover, any electrical source resistance can be used for exciting the cMUT. Hence, the electrical mismatch between the electrical source and the cMUT is unimportant. In this case, it is reasonable to try to maximize the pressure at the mechanical side while the maximum allowed voltage is applied at the electrical port. Referring to Fig. 2(a), let $P$ be the pressure in the immersion medium, $P=F / S$, when the applied alternating current (AC) voltage, $V_{S}$, is at the maximum allowable value. $B_{1}$ is the associated $3 \mathrm{~dB}$ bandwidth of the output pressure. In the transmission mode, we define the figure of merit as the pressure-bandwidth product:

$$
M_{T}=P B_{1} .
$$

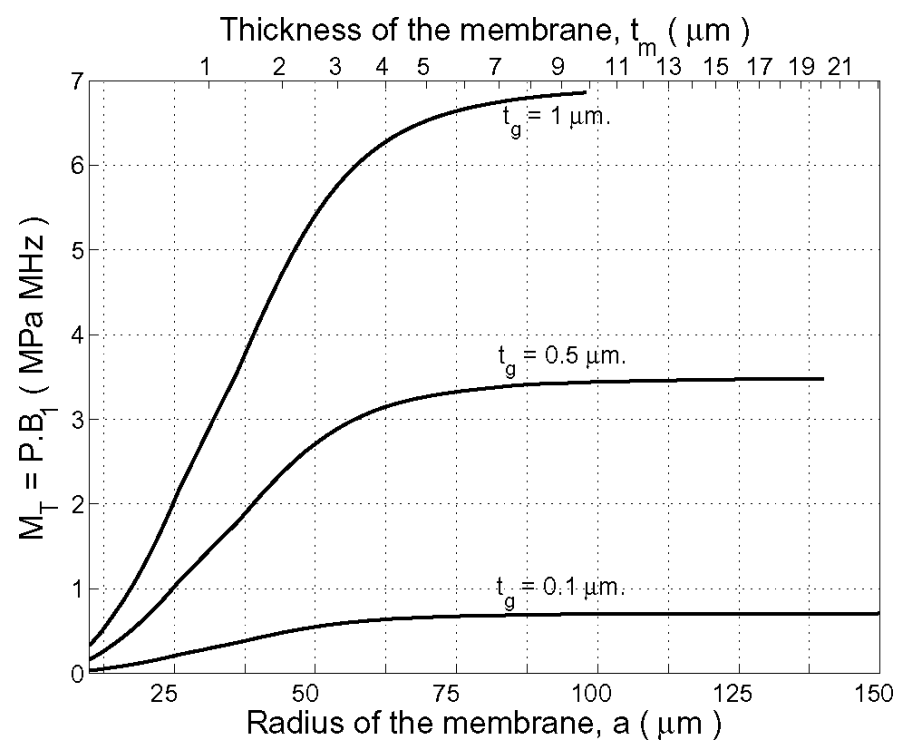

Fig. 3. Pressure-bandwidth product, $M_{T}$, of a cMUT resonating at $5 \mathrm{MHz}$ and operating as a transmitter in water as a function of membrane radius, $a$ (or as a function of membrane thickness, $t_{m}$ ) for different gap heights. $t_{m} / a^{2}$ is kept constant. The bias voltage is $V_{D C}=0.45 V_{\text {col }}$ and the electrical source resistance, $R_{S}$, is zero.

A calculation ${ }^{3}$ of $M_{T}$ is done using the corrected Mason model. If the maximum peak voltage on the electrode is $0.9 V_{c o l}, n_{c}$ is calculated from (6) with $V_{D C}=0.45 V_{c o l}$. Although the cMUT is highly nonlinear with a large excitation, we treat the problem as if it is linear for simplicity, and $n_{c}$ is assumed to be independent of the applied AC voltage. The resulting $M_{T}$ is seen in Fig. 3 as a function of $a$ or $t_{m}$ with the gap height, $t_{g}$, as a parameter. In order to have the same membrane resonance, $t_{m} / a^{2}$ is kept constant as $a$ or $t_{m}$ is varied. In Fig. 4, the resulting bandwidth, $B_{1}$, and 3 -dB lower corner frequency, $f_{1}$, are plotted. ( $3 \mathrm{~dB}$ band extends from $f_{1}$ to $f_{1}+B_{1}$.)

We see that larger radii (or thicker membranes) give higher pressure-bandwidth products, but smaller bandwidths. For higher bandwidth values, the pressurebandwidth product must be sacrificed. In other words, large bandwidth values are possible with only very small gain values. In all cases, larger gap heights are preferable because the corresponding collapse voltages are higher. With a higher applied input voltage, a higher pressure is possible. Bandwidth $B_{1}$ is found to be independent of the gap height.

\section{B. Receive Mode}

Unlike the transmission mode in which we may have unlimited electrical input power, in receive mode the input acoustic power is limited. It is important to use as much of the available acoustic power as possible. For the best performance, the acoustic mismatch at the mechanical side should be minimized. Similarly, the electrical mismatch at

\footnotetext{
${ }^{3}$ MATLAB (The MathWorks Inc., Natick, MA) is used to evaluate the expressions. Scattering parameters of the Mason's model are determined using the equations given in p. 210 of [22].
} 


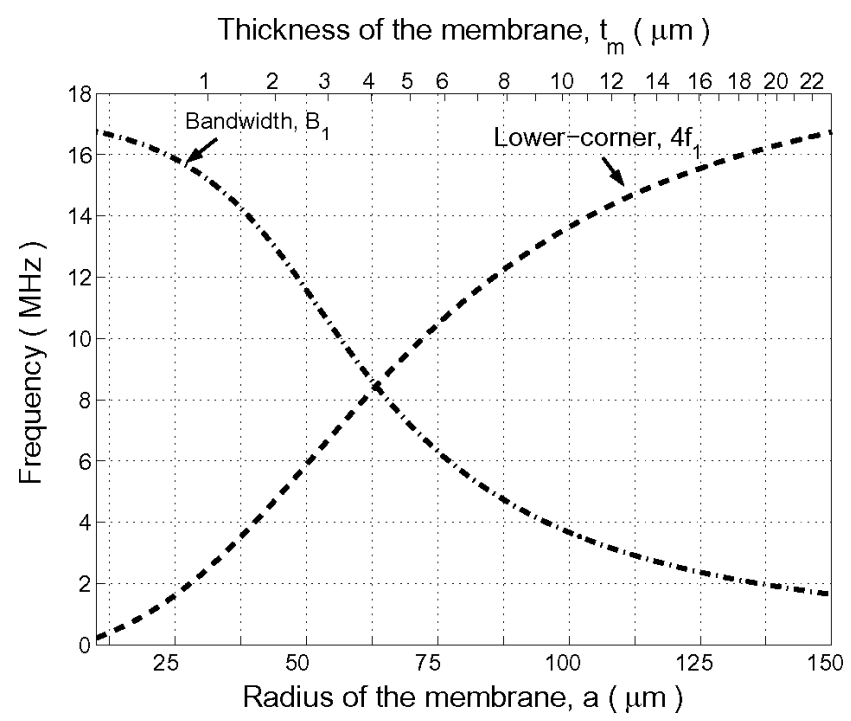

Fig. 4. Bandwidth (dash-dot), $B_{1}$, and lower corner frequency (dashed), $f_{1}$, of a cMUT resonating at $5 \mathrm{MHz}$ and operating as a transmitter in water as a function of membrane radius, $a$, (or as a function of membrane thickness, $\left.t_{m}\right) \cdot t_{m} / a^{2}$ is kept constant. $B_{1}$ and $f_{1}$ are independent of $t_{g} . f_{1}$ curve is multiplied by four to improve readability. The bias voltage is $V_{D C}=0.45 V_{c o l}$, and the electrical source resistance, $R_{S}$, is zero.

the electrical side should be kept at a minimum for good performance. Mismatch loses at both sides are included, if we use the transducer gain definition [Refer to Fig. 2(b)]:

$$
G_{T}=P_{E} / P_{A},
$$

where $P_{E}$ is the power delivered to the electrical load resistance, $R_{S}$, and $P_{A}$ is the available acoustic power ${ }^{4}$ from the immersion medium. The highest transducer gain is obtained if the electrical side impedance of the transducer is conjugately matched to the receiver impedance, and the acoustic side impedance of the transducer is equal to the acoustic impedance of the immersion medium. Because the transducer gain is a power gain, we define the gain as the square root of the transducer gain and the bandwidth, $B_{2}$, as the $3 \mathrm{~dB}$ bandwidth of the transducer gain. Hence, in the receive mode, we define a figure of merit, $M_{R}$, as the gain-bandwidth product:

$$
M_{R}=\sqrt{G_{T}} B_{2} .
$$

In what follows, we will investigate the effect of various parameters on this product. We have determined that the gap height does not affect $M_{R}$, provided that the cMUT is biased with the same percentage value of the collapse voltage. For all cases we keep the bias voltage at $V_{D C}=$ $0.9 V_{\text {col }}$.

We calculated and plotted $M_{R}$ as a function of $a$ or $t_{m}$ in Fig. 5 for the cMUTs immersed in water. We note that the electrical termination resistance, $R_{S}$, is optimally

\footnotetext{
${ }^{4}$ Available power is the power delivered to a load when the load impedance is conjugately matched to the source impedance (see p. 610 of $[22])$.
}

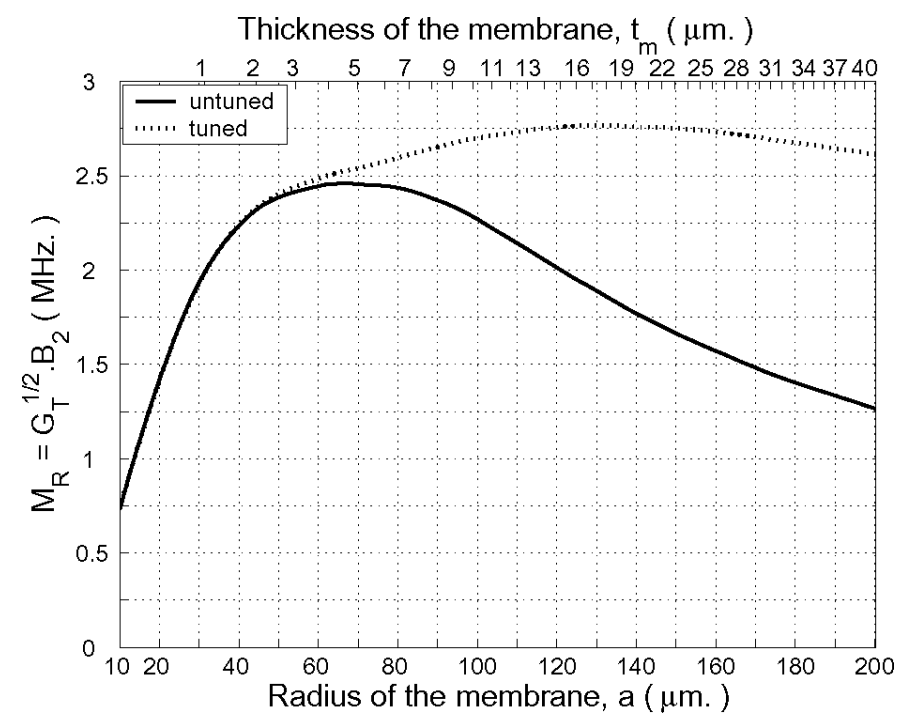

Fig. 5. Gain-bandwidth product, $M_{R}$, of water-immersed receiving mode cMUTs resonating at $5 \mathrm{MHz}$ as a function of membrane radius, $a$, or membrane thickness, $t_{m}$, for untuned (solid) and tuned (dotted) cases. $t_{m} / a^{2}$ is kept constant. Electrical termination resistance, $R_{S}$, is optimal at every point. $V_{D C}=0.9 V_{c o l}$. The curves are independent of the gap height.

chosen $^{5}$ for each $a-t_{m}$ pair. For the membranes resonating at $5 \mathrm{MHz}$, the highest gain-bandwidth product is obtained for $a=70 \mu \mathrm{m}$ and $t_{m}=5 \mu \mathrm{m}$. If a shunt tuning inductor is added at the electrical port, a further improvement in the gain-bandwidth product is possible as shown in Fig. 5 . The value of this inductor is chosen to maximize the gainbandwidth product. In this case, $a=130 \mu \mathrm{m}, t_{m}=18 \mu \mathrm{m}$ and $L_{T}=1.5 \mu \mathrm{H}$ gives the best $M_{R}$.

For small $a$ values, $Z_{m} S$ is negligible compared to $Z_{a} S$, and the equivalent circuit may be simplified to an RC circuit. In this case, the tuning does not bring any improvement. But when the mechanical impedance of the membrane is significant, an inductor provides a better match at the electrical port.

The tradeoff between the gain and the bandwidth is demonstrated graphically in Fig. 6 as a function of $a$ or $t_{m}$. As $a$ goes up, the bandwidth decreases and the gain increases. We note that, for each radius value, a different membrane thickness is used in such a way to keep the membrane resonance at $5 \mathrm{MHz}$. In the same figure, the effect of tuning is also indicated. It is clear that adding an inductor does not have a positive effect on the bandwidth; hence, it should be used only when a higher gain is a necessity.

We demonstrate the effect of the electrical termination resistance on the gain-bandwidth product in Figs. 7 and 8. It is obvious that there is an optimum $R_{S}$ value to maximize the gain-bandwidth product. Because $n_{c}$ depends on the bias voltage, the optimum $R_{S}$ will be different for different gap heights. We note that in Fig. 7 the given $R_{S}$ is for one cMUT unit cell. If the actual electrical termi-

\footnotetext{
${ }^{5} R_{S}$ value is found by a binary search conducted in a range of resistance values for all $a-t_{m}$ pairs.
} 


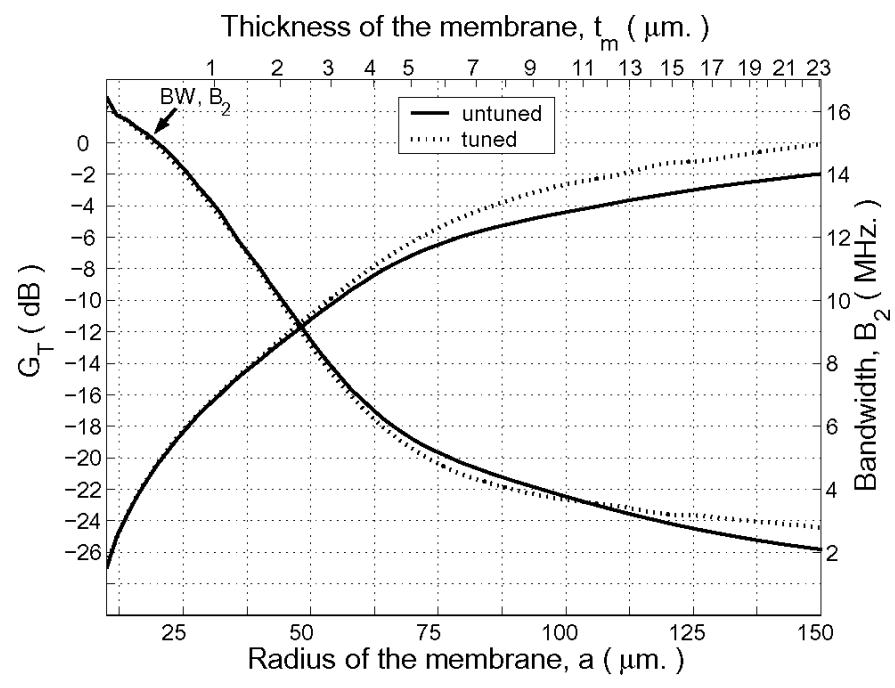

Fig. 6. Dependence of gain and bandwidth on the membrane radius or thickness for untuned (solid) and tuned (dotted) cMUTs immersed in water and resonating at $5 \mathrm{MHz} . R_{S}$ is optimal at every point $\left(V_{D C}=0.9 V_{c o l}\right)$. The curves are independent of the gap height.

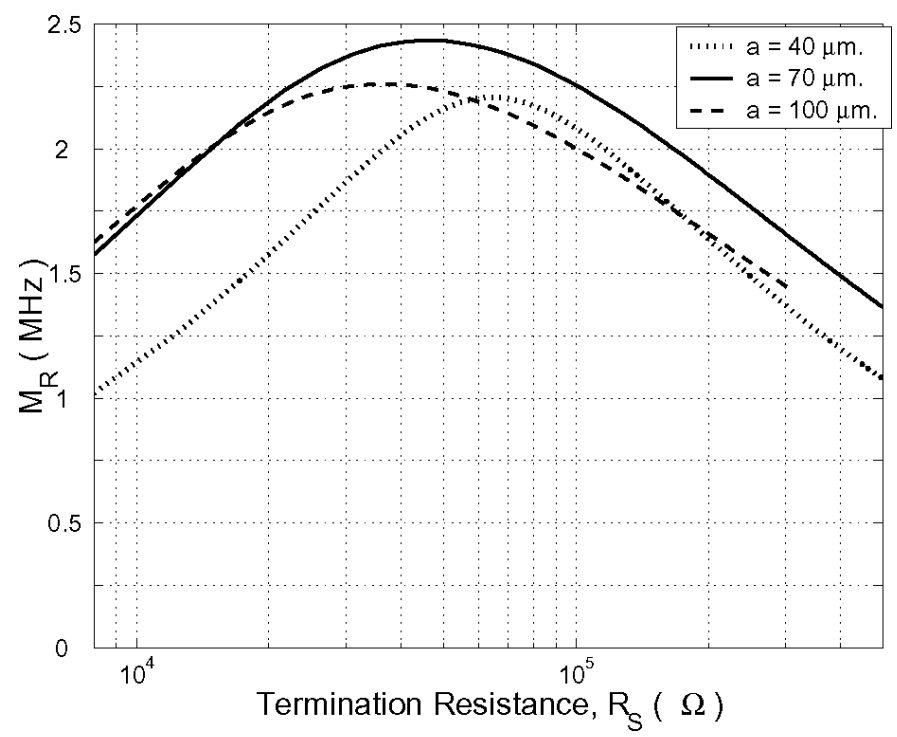

Fig. 7. Effect of electrical termination resistance, $R_{S}$, on the gainbandwidth product for different cMUTs resonating at $5 \mathrm{MHz}$ immersed in water. $\left(t_{g}=0.3 \mu \mathrm{m}, V_{D C}=0.9 V_{c o l}\right)$

nation resistance value, $R_{S a}$, is lower, we need to connect $N=R_{S} / R_{S a}$ many cMUTs in parallel to achieve the desired match. For example, in Fig. 7 a cMUT with $a=70 \mu \mathrm{m}$ requires an $R_{S}$ of approximately $50 \mathrm{k} \Omega$ for maximum $M_{R}$, and if 100 such cMUTs are in parallel, an electrical load of $500 \Omega$ is necessary. Changing the value of $R_{S}$ is a very simple way of trading gain with bandwidth at the expense of some loss in the gain-bandwidth product. Referring to $a=70 \mu \mathrm{m}$ curve in Fig. 7 and Fig. 8, we notice that, although $R_{S}$ is reduced by a factor of five from its optimal value, we lose the gain by a factor of two $(6 \mathrm{~dB})$, but the bandwidth can be increased only by $32 \%$.

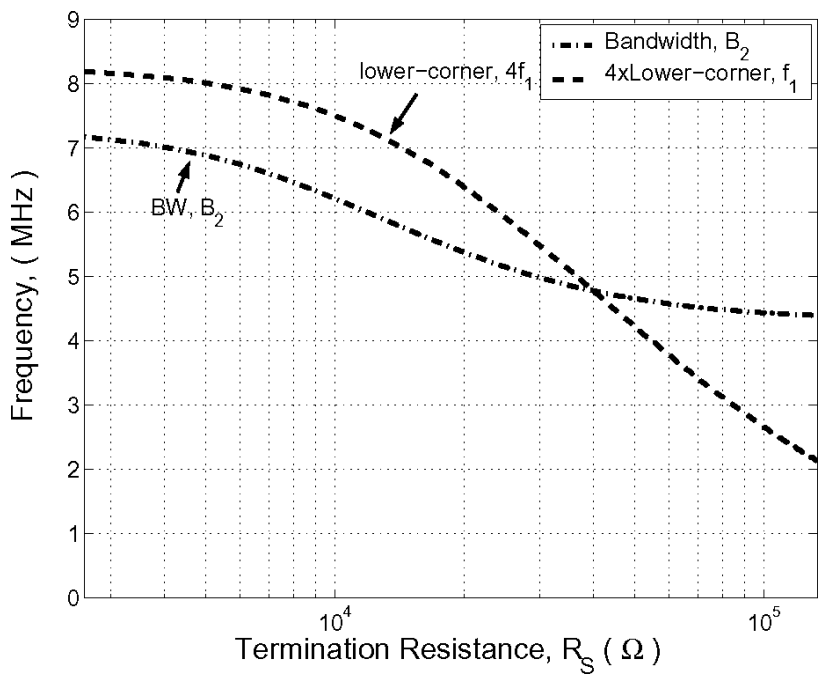

Fig. 8. Effect of electrical termination resistance, $R_{S}$, on the bandwidth (dash-dot) and lower corner frequency (dash) for a cMUT with $70 \mu \mathrm{m}$. radius resonating at $5 \mathrm{MHz}$ immersed in water. $\left(t_{g}=0.3 \mu \mathrm{m}\right.$, $\left.V_{D C}=0.9 V_{c o l}\right)$

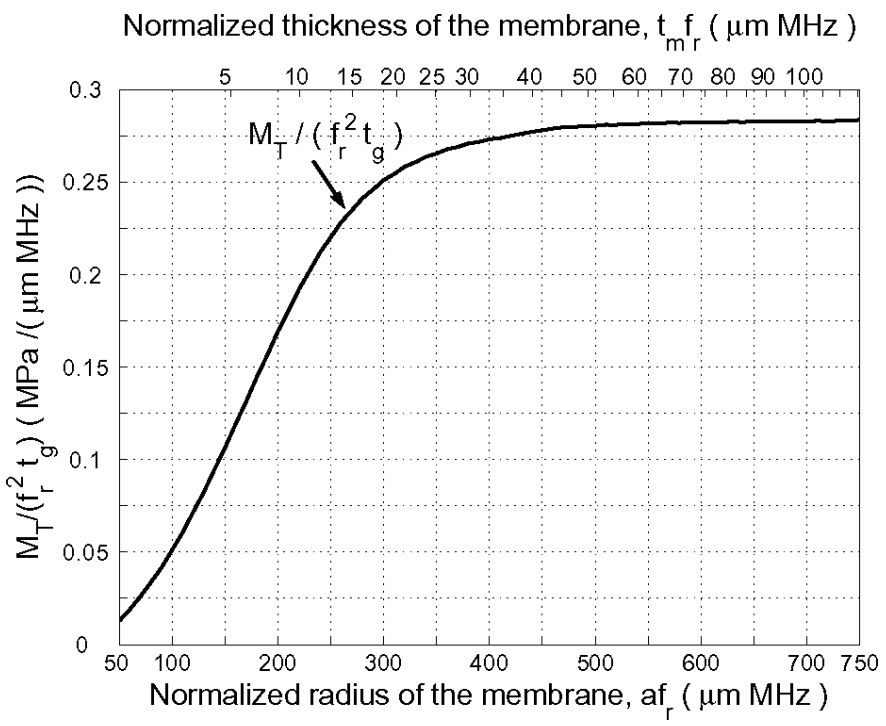

Fig. 9. Normalized pressure-bandwidth product as a function of normalized membrane radius or thickness for transmitter cMUTs. Bias voltage is at $45 \%$, and applied peak-to-peak AC voltage is at $90 \%$ of the collapse voltage.

\section{Pulse-Echo Mode}

In most applications the same transducer is used for both transmission and receive, and it is operated in the pulse-echo mode. A transmit-receive switch connects either the transmitter amplifier or the receiver circuit to the electrical side of the cMUT, depending on the mode of operation. Hence, the electrical termination resistance, $R_{S}$, can be different for transmit and receive modes. In this case, a figure of merit can be defined as:

$$
M_{P E}=P \sqrt{G_{T}} B_{3},
$$




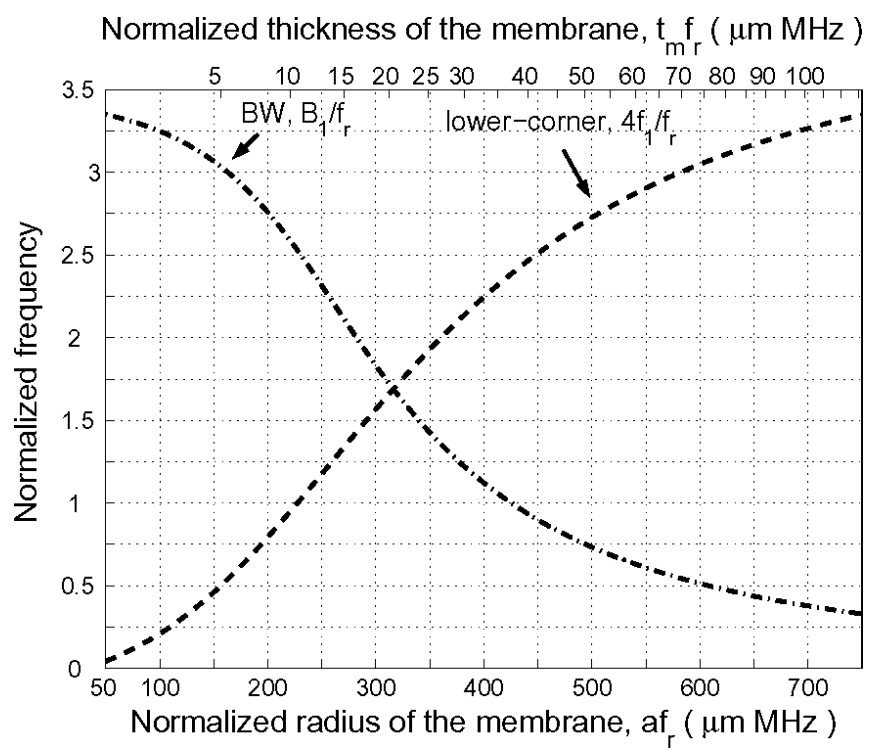

Fig. 10. Normalized bandwidth (dash-dot) and lower corner frequency (dashed) as a function of normalized membrane radius or thickness for transmitter cMUTs. Bias voltage is at $45 \%$, and applied peak-to-peak AC voltage is at $90 \%$ of the collapse voltage.

where $P$ is defined as in (9), $G_{T}$ is defined as in (10), and $B_{3}$ is the $3 \mathrm{~dB}$ bandwidth of the $P \sqrt{G_{T}}$ product.

\section{Design Graphs}

In what follows, we will present normalized versions of the graphs that can be used as design tools for cMUTs with silicon nitride membranes. A number of examples are given to demonstrate the use of these graphs.

\section{A. Transmit Mode}

Figs. 9, 10, and 11 are normalized graphs that can be used to determine the dimensions of a transmitter cMUT at specified frequencies. The first two are essentially the same graphs as Figs. 3 and 4 with its axes normalized with respect to resonance frequency and gap height ${ }^{6}$. Notice that all axes are normalized and their relation with the actual values is provided in the axis labels. Let us demonstrate the use of the graphs by designing a transmitter cMUT to operate between 3 - $\mathrm{dB}$ frequencies $f_{1}$ to $f_{2}$ with an output pressure as high as possible. Suppose $f_{1}=3 \mathrm{MHz}$ and $f_{2}=20 \mathrm{MHz}$. We start at a point with a high $M_{T}$ such as $a f_{r}=300$. At this point we read from Fig. $10 B_{1} / f_{r}=1.8$. Because we need a bandwidth of $f_{2}-f_{1}=B_{1}=17 \mathrm{MHz}$, resonance frequency should be $f_{r}=17 / 1.8=9.3 \mathrm{MHz}$. The lower corner $\left(f_{1}\right)$ of the band can be determined from Fig. 10 as $4 f_{1} / f_{r}=1.55$ or $f_{1}=3.6 \mathrm{MHz}$. Because this is larger than the required $3 \mathrm{MHz}$, we need more iterations. $a f_{r}=285$ gives

\footnotetext{
${ }^{6}$ Because of the fringe field extension of the radius to $a+\bar{t}_{g}$ in (5), there is a difficulty of normalization with respect to $t_{g}$. However, the graphs remain valid as long as $t_{g} \ll a$.
}

Normalized thickness of the membrane, $\mathrm{t}_{\mathrm{m}} \mathrm{f}_{\mathrm{r}}(\mu \mathrm{m} \mathrm{MHz})$

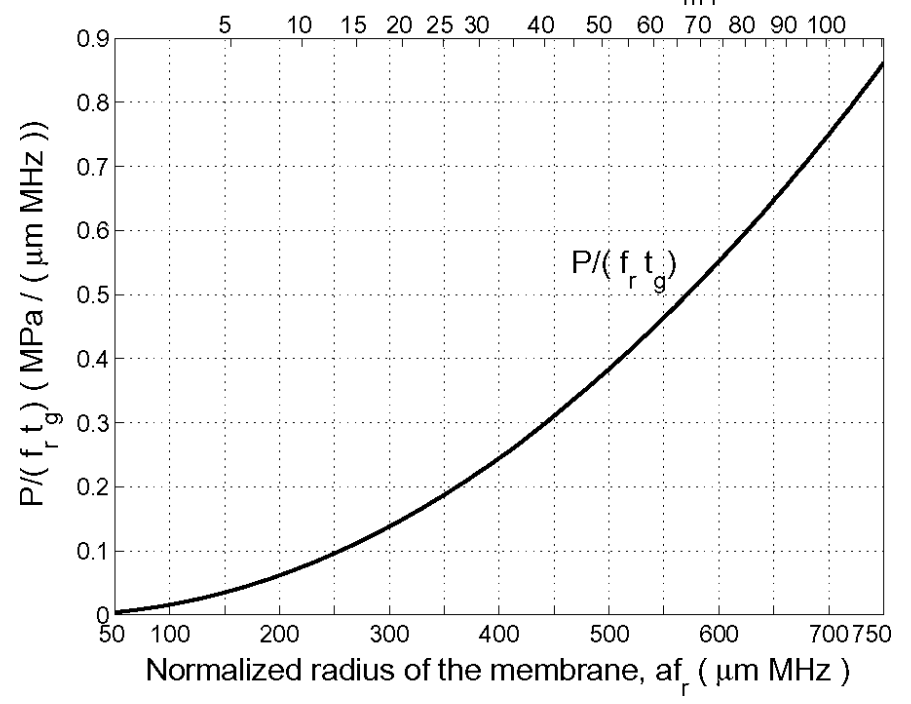

Fig. 11. Normalized pressure as a function of normalized membrane radius or thickness for transmitter cMUTs. Bias voltage is at $45 \%$, and applied peak-to-peak AC voltage is at $90 \%$ of the collapse voltage.

satisfactory results. We find $f_{r}=17 / 2=8.5 \mathrm{MHz}$, $f_{1}=8.5 \times 1.4 / 4 \simeq 3 \mathrm{MHz}$ and $a=34 \mu \mathrm{m}$. We determine from the upper x-axis of Fig. $10 t_{m} f_{r}=17$ or $t_{m}=17 / 8.5=2 \mu \mathrm{m}$. We should pick a collapse voltage as high as possible. Let $V_{c o l}=150 \mathrm{~V}$. Eq. (8) gives nearly the same result as the method in [12]: $\bar{t}_{g}$ should be $0.35 \mu \mathrm{m}$. To make sure that $150 \mathrm{~V}$ does not cause a breakdown of the nitride stand, we calculate the E-field: $150 / 0.35=428 \mathrm{~V} / \mu \mathrm{m}$ which is well below the breakdown voltage. Pressurebandwidth product, $M_{T}$, is determined from Fig. 9 as $M_{T}=0.24 \times 8.5^{2} \times 0.35 \simeq 6 \mathrm{MPa}-\mathrm{MHz}$. Hence the output pressure corresponding to an excitation voltage of $0.9 \times 150=135 \mathrm{~V}$ peak-to-peak is $P=6 / 17 \simeq 0.35 \mathrm{MPa}$. To verify results we performed FEM simulations of the same structure resulting in $f_{1}=2.8 \mathrm{MHz}, B_{1}=16 \mathrm{MHz}$, $V_{c o l}=153 \mathrm{~V}, P=0.33 \mathrm{MPa}$ and $M_{T}=5.37$.

As a second example, suppose we need a cMUT with an output pressure of $P=0.5 \mathrm{MPa}$ at a center frequency of $8 \mathrm{MHz}$. Let us determine the dimensions. With a reasonable gap height of $t_{g}=0.2 \mu \mathrm{m}$ and $f_{r}=8 \mathrm{MHz}$ we find $P /\left(f_{r} t_{g}\right)=0.5 /(8 \times 0.2)=0.32$ and from Fig. 11 we determine $a f_{r}=450$ and $t_{m} f_{r}=42$ or $a=56 \mu \mathrm{m}$ and $t_{m}=5.2 \mu \mathrm{m}$. We estimate $V_{c o l}=101 \mathrm{~V}$ from (8). From Fig. 10 we find the bandwidth $B_{1}=0.8 \times 8=6.4 \mathrm{MHz}$ and $f_{1}=2.5 \times 8 / 4=5 \mathrm{MHz}$. Hence, the center frequency is at $5+6.4 / 2=8.2 \mathrm{MHz}$. FEM simulations for the given parameters produce $f_{1}=4.6 \mathrm{MHz}, B_{1}=7.2 \mathrm{MHz}$, $V_{c o l}=109 \mathrm{~V}$ and $P=0.48 \mathrm{MPa}$.

\section{B. Receive Mode}

Normalized graphs to design receiving mode cMUTs are shown in Figs. 12, 13, and 14. There is no tuning induc- 
Normalized thickness of the membrane, $\mathrm{t}_{\mathrm{m}} \mathrm{f}_{\mathrm{r}}(\mu \mathrm{m} \mathrm{MHz})$

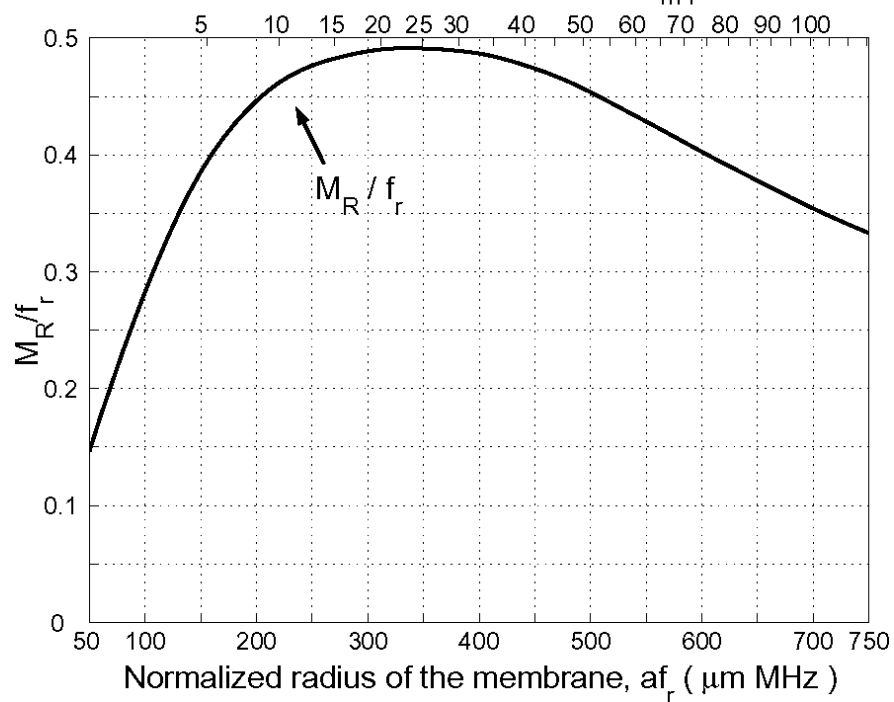

Fig. 12. Normalized gain-bandwidth product as a function of normalized membrane radius or thickness for receiver cMUTs without tuning. The curve is independent of the gap height.

tance, but the electrical load resistance, $R_{S}$, is chosen at the value to maximize the gain-bandwidth product.

As an example of use of these graphs, suppose we need a receiver cMUT with $B_{2}=14 \mathrm{MHz}$ of bandwidth between $f_{1}=1 \mathrm{MHz}$ and $f_{2}=15 \mathrm{MHz} 3 \mathrm{~dB}$ corner frequencies. At $a f_{r}=350$, we read $B_{2} / f_{r} \simeq 1.1$ from Fig. 13 and determine $f_{r}=12.5 \mathrm{MHz}$. For this choice, we use the $f_{1}$ curve in Fig. 13 and find $5 f_{1} / f_{r} \simeq 1.7$. So, we calculate $f_{1}=4.3 \mathrm{MHz}$, which does not satisfy our requirement of $1 \mathrm{MHz}$ for the lower end frequency. After a few iterations, we find that $a f_{r}=200$ and $f_{r}=6.4 \mathrm{MHz}$ give satisfactory results. Hence, $a=31.5 \mu \mathrm{m}$ and $t_{m}=8.3 / 6.4=1.3 \mu \mathrm{m}$. The gain-bandwidth product is determined from Fig. 12 as $M_{R} / f_{r} \simeq 0.45$ or $M_{R}=2.8 \mathrm{MHz}$. Therefore, the transducer power gain of the cMUT is $\sqrt{G_{T}}=2.8 / 14=0.2=$ $-14 \mathrm{~dB}$. The gap height does not affect the performance, and it should be chosen to give an acceptable bias voltage. For example, $t_{g}=0.3 \mu \mathrm{m}$ gives $V_{c o l}=74 \mathrm{~V}$. FEM simulations of the cMUT with the dimensions above give a bandwidth of $13.7 \mathrm{MHz}$ starting at $f_{1}=940 \mathrm{KHz}$ with $V_{c o l}=76 \mathrm{~V}$ and $G_{T}=-13.4 \mathrm{~dB}$ verifying the predicted gain and bandwidth values.

As a further example, suppose we need to design a cMUT with a transducer gain of $-3 \mathrm{~dB}$ centered at $10 \mathrm{MHz}$. From Fig. 14 we find $a f_{r}=610 \mu \mathrm{m}-\mathrm{MHz}$ or $t_{m} f_{r}=75 \mu \mathrm{mMHz}$ satisfies the gain requirement. We also find from Fig. $13 B_{2} / f_{r}=0.6$ and $5 f_{1} / f_{r}=3.1$. To make $f_{1}+B_{2} / 2=10 \mathrm{MHz}$ we set $3.1 f_{r} / 5+0.6 f_{r} / 2=10 \mathrm{MHz}$ or $f_{r}=10.9 \mathrm{MHz}, f_{1}=6.7 \mathrm{MHz}, B_{2}=6.5 \mathrm{MHz}$. Hence, $a=56 \mu \mathrm{m}$ and $t_{m}=6.9 \mu \mathrm{m}$. Because this is a rather thick membrane, the gap should be very small to give an acceptable collapse voltage. For $t_{g}=0.1 \mu \mathrm{m}$ we find $V_{c o l}=57 \mathrm{~V}$. On the other hand, the values determined from FEM are: $f_{1}=5.7 \mathrm{MHz}, B_{2}=6.5 \mathrm{MHz}, V_{\text {col }}=65 \mathrm{~V}, G_{T}=-2.8 \mathrm{~dB}$.
Normalized thickness of the membrane, $\mathrm{t}_{\mathrm{m}_{\mathrm{r}}}(\mu \mathrm{m} \mathrm{MHz})$

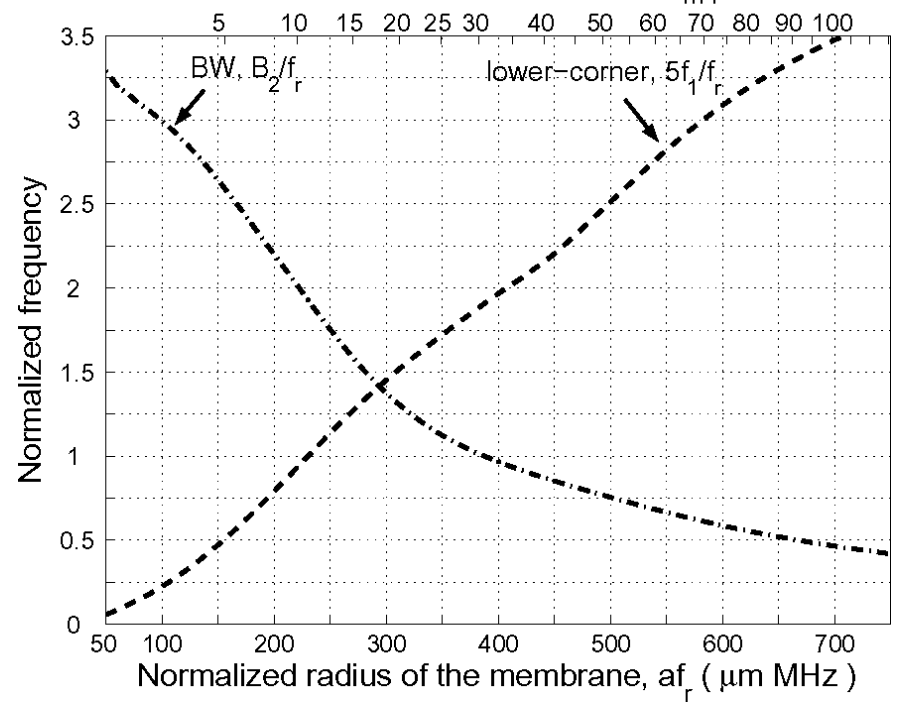

Fig. 13. Normalized bandwidth (dash-dot) and lower corner frequency (dashed) as a function of normalized membrane radius or thickness for receiver cMUTs without tuning. The curves are independent of the gap height.

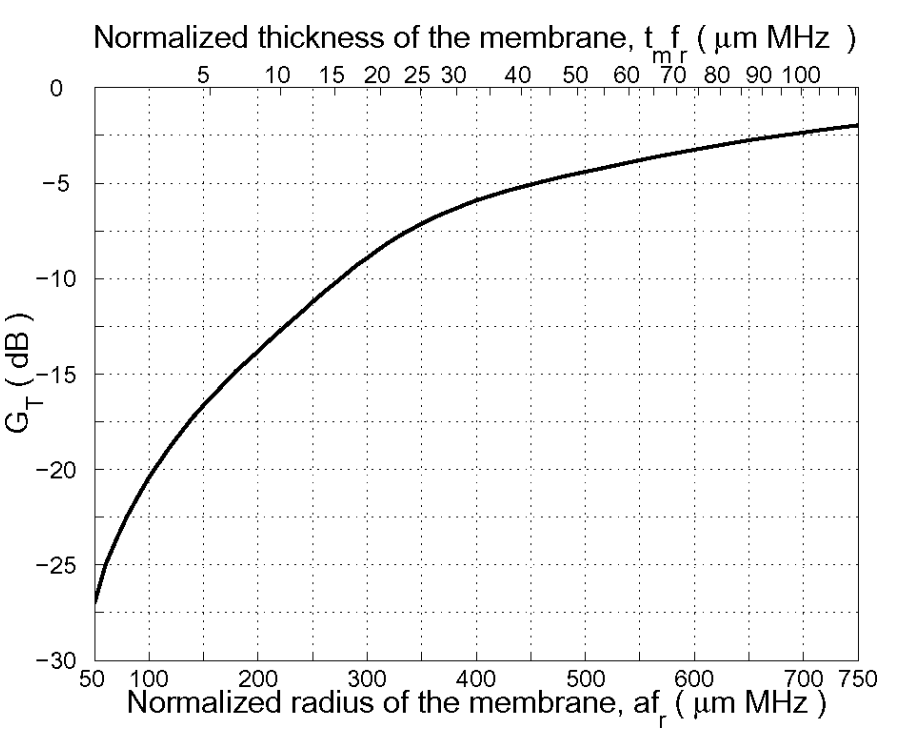

Fig. 14. Normalized transducer gain as a function of normalized membrane radius or thickness for receiver cMUTs without tuning.

\section{Pulse-Echo Mode}

Figs. 15 and 16 also are normalized graphs that can be used to design cMUTs in pulse-echo mode in a similar manner. Inspection of the first graph shows that one should prefer larger gap heights for the best figure of merit. Although a larger membrane radius gives a better merit figure, it results in a smaller bandwidth. As an example, we design a transducer with an overall bandwidth of $B_{3}=14 \mathrm{MHz}$ between $3 \mathrm{~dB}$ corner frequencies of $1 \mathrm{MHz}$ and $15 \mathrm{MHz}$. We find from Fig. 16 by iteration at $a f_{r}=160 \mu \mathrm{m} \mathrm{MHz}, B_{3}=2.3 f_{r}$, and $f_{1}=0.7 f_{r} / 4$, resulting in $f_{r}=14 / 2.3=6 \mathrm{MHz}$ and $f_{1}=1 \mathrm{MHz}$. Hence, 


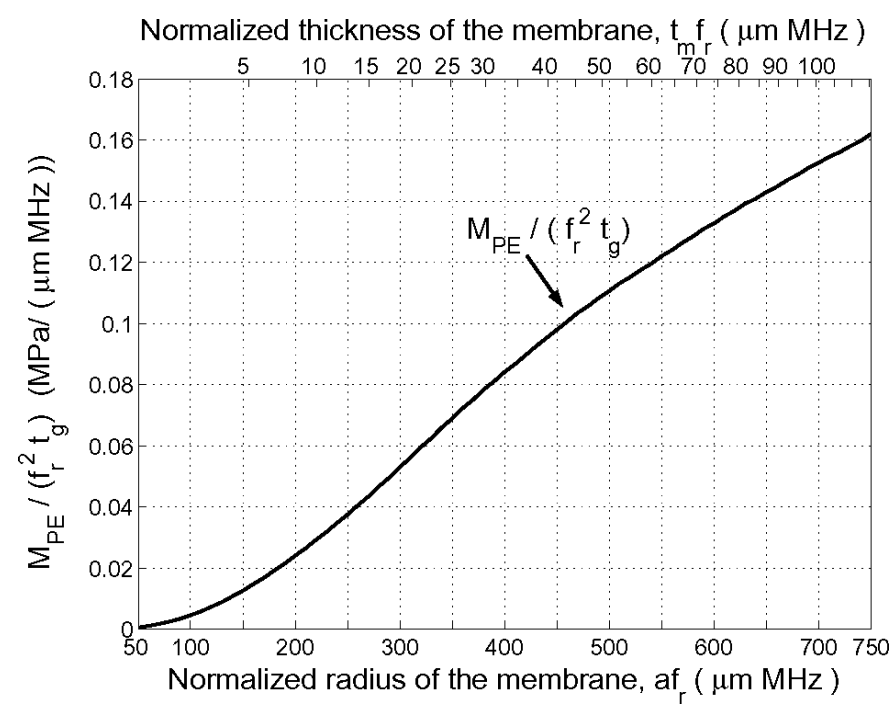

Fig. 15. Normalized pressure-gain-bandwidth product as a function of normalized membrane radius or thickness for cMUTs in pulse-echo mode. Bias voltage for transmit is at 45\%, and applied peak-to-peak $\mathrm{AC}$ voltage is at $90 \%$ of the collapse voltage. Bias voltage for receive is at $90 \%$ of the collapse voltage.
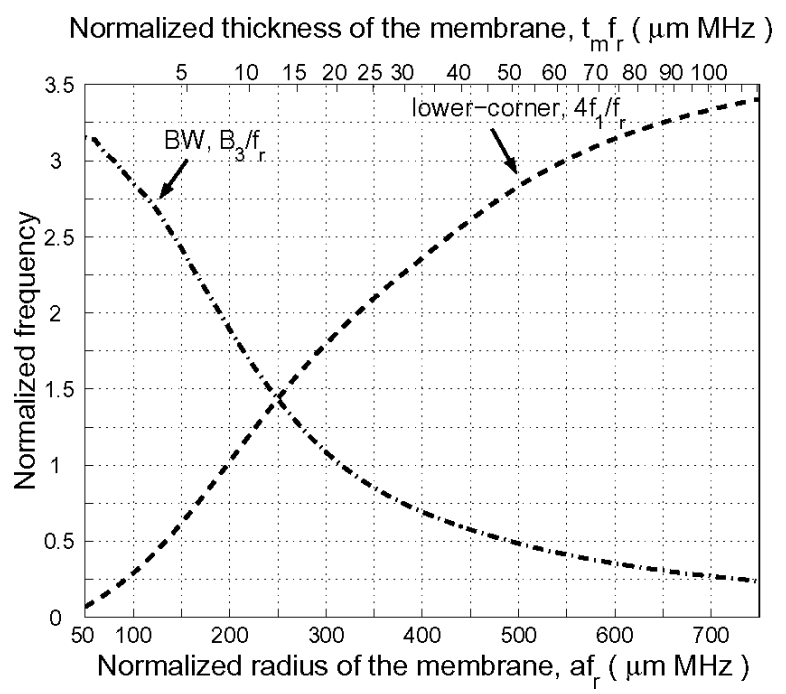

Fig. 16. Normalized overall bandwidth (dash-dot), and lower corner frequency (dashed) as a function of normalized membrane radius or thickness for cMUTs in pulse-echo mode.

$a=160 / 6=27 \mu \mathrm{m}, t_{m}=5.2 / 6=0.9 \mu \mathrm{m}$ are determined. If $V_{c o l}=50 \mathrm{~V}$, we find from (8) $\bar{t}_{g}=0.27 \mu \mathrm{m}$. In transmitter mode we find from Fig. $10 B_{1}=18 \mathrm{MHz}$ and from Fig. $11 P=0.065 \mathrm{MPa}$. In receive mode we use Fig. 13 to find $B_{2}=16 \mathrm{MHz}$ and Fig. 14 to find $G_{T}=-16 \mathrm{~dB}$.

\section{Conclusions}

We defined performance measures for cMUTs in transmit, receive, or pulse-echo modes and described the ways of determining the optimum dimensions. In transmit and pulse-echo modes, cMUTs with large gaps are preferable because the collapse voltages are higher; hence, higher excitation voltages are possible. In general, there is a tradeoff between bandwidth and gain-bandwidth product. Smaller membrane radii result in higher bandwidth at the expense of a reduced gain-bandwidth product. For the cMUTs operating in receive mode, the gap height does not affect the figure of merit if cMUT is biased at the same percentage value of the collapse voltage. There is an optimal value of the membrane radius or thickness and an optimal electrical termination resistance for the highest gain-bandwidth product. One should sacrifice some gain-bandwidth product if a higher bandwidth is necessary.

We introduced design tools to determine approximately the optimum dimensions of the cMUTs with given frequency response. Our results could be on the optimistic side because we did not include the effect of spurious capacitors. One should use a full FEM analysis, including the liquid loading, if more accurate results are desired.

\section{REFERENCES}

[1] H. T. Soh, I. Ladabaum, A. Atalar, C. F. Quate, and B. T. Khuri-Yakub, "Silicon micromachined ultrasonic immersion transducers," Appl. Phys. Lett., vol. 69, pp. 3674-3676, 1996.

[2] I. Ladabaum, X. Jin, H. T. Soh, F. Pierre, A. Atalar, and B. T. Khuri-Yakub, "Microfabricated ultrasonic transducers: Towards robust models and immersion devices," in Proc. IEEE Ultrason. Symp., 1996, pp. 335-338.

[3] A. Burhdorf, O. Ahrens, and J. Binder, "Capacitive micromachined ultrasonic transducers and their application," in Proc. IEEE Ultrason. Symp., 2001, pp. 933-940.

[4] J. S. McIntosh, D. A. Hutchins, G. Etcheverry, D. R. Billson, R. A. Noble, R. R. Davies, and L. Koker, "Micromachined capacitive transducer array for imaging in air," in Proc. IEEE Ultrason. Symp., 2001, pp. 929-932.

[5] J. S. McIntosh, D. A. Hutchins, D. R. Billson, T. J. Robertson, R. A. Noble, and A. D. R. Jones, "The characterization of capacitive micromachined ultrasonic transducers in air," Ultrasonics, vol. 40, pp. 477-483, 2002.

[6] J. Johnson, Ö. Oralkan, U. Demirci, S. Ergun, M. Karaman, and B. T. Khuri-Yakub, "Medical imaging using capacitive micromachined ultrasonic transducer arrays," Ultrasonics, vol. 40, pp. 471-476, 2002.

[7] Ö. Oralkan, A. S. Ergun, J. A. Johnson, M. Karaman, U. Demirci, K. Kaviani, T. H. Lee, and B. T. Khuri-Yakub, "Capacitive micromachined ultrasonic transducers: Next generation arrays for acoustic imaging?," in IEEE Trans. Ultrason., Ferroelect., Freq. Contr., vol. 49, pp. 1596-1610, 2002.

[8] Ö. Oralkan, A. S. Ergun, C.-H. Cheng, J. A. Johnson, M. Karaman, T. H. Lee, and B. T. Khuri-Yakub, "Volumetric ultrasound imaging using 2-D cMUT arrays," in IEEE Trans. Ultrason., Ferroelect., Freq. Contr., vol. 50, pp. 1581-1594, 2003.

[9] A. Bozkurt, F. L. Degertekin, A. Atalar, and B. T. Khuri-Yakub, "Analytic modelling of loss and cross-coupling in capacitive micromachined ultrasonic transducers," in Proc. IEEE Ultrason. Symp., 1998, pp. 1025-1028.

[10] I. Ladabaum, X. Jin, H. T. Soh, A. Atalar, and B. T. KhuriYakub, "Surface micromachined capacitive ultrasonic transducers," IEEE Trans. Ultrason., Ferroelect., Freq. Contr., vol. 45, pp. 678-690, 1998.

[11] A. Caronti, G. Caliano, A. Iula, and M. Pappalardo, "An accurate model for capacitive micromachined ultrasonic transducers," IEEE Trans. Ultrason., Ferroelect., Freq. Contr., vol. 49, pp. 159-167, 2002.

[12] A. Nikoozadeh, B. Bayram, G. G. Yaralioglu, and B. T. KhuriYakub, "Analytical calculation of collapse voltage of cMUT membrane," in Proc. IEEE Ultrason. Symp., 2004, pp. 256-259.

[13] Y. Roh and B. T. Khuri-Yakub, "Finite element analysis of underwater capacitor micromachined ultrasonic transduc- 
ers," IEEE Trans. Ultrason., Ferroelect., Freq. Contr., vol. 49, pp. 293-298, 2002.

[14] A. Bozkurt, I. Ladabaum, A. Atalar, and B. T. Khuri-Yakub, "Theory and analysis of electrode size optimization for capacitive microfabricated ultrasonic transducers," IEEE Trans. Ultrason., Ferroelect., Freq. Contr., vol. 46, pp. 1364-1374, 1999.

[15] J. McLean, R. O. Guldiken, and F. L. Degertekin, "CMUTs with dual electrode structure for improved transmit and receive performance," in Proc. IEEE Ultrason. Symp., 2004, pp. 501504.

[16] B. Bayram, E. Hæggström, G. G. Yaralioglu, and B. T. KhuriYakub, "A new regime for operating capacitive micromachined ultrasonic transducers," IEEE Trans. Ultrason., Ferroelect., Freq. Contr., vol. 50, pp. 1184-1190, 2003.

[17] G. G. Yaralioglu, M. H. Badi, A. S. Ergun, and B. T. KhuriYakub, "Improved equivalent circuit and finite element method modelling of capacitive micromachined ultrasonic transducers," in Proc. IEEE Ultrason. Symp., 2003, pp. 469-472.

[18] B. T. Khuri-Yakub, C. Cheng, F. L. Degertekin, S. Ergun, S. Hanse, X. C. Jin, and Ö. Oralkan, "Silicon micromachined ultrasonic transducers," Jpn. J. Appl. Phys., vol. 39, pp. 2883-2887, 2000.

[19] G. G. Yaralioglu, A. S. Ergun, B. Bayram, E. Hæggström, and B. T. Khuri-Yakub, "Calculation and measurement of electromechanical coupling coefficient of capacitive micromachined ultrasonic transducers," IEEE Trans. Ultrason., Ferroelect., Freq. Contr., vol. 50, pp. 449-456, 2003.

[20] U. Demirci, J. A. Johnson, A. S. Ergun, M. Karaman, and B. T. Khuri-Yakub, "Capacitive micromachined ultrasonic transducer arrays for medical imaging: Experimental results," in Proc. IEEE Ultrason. Symp., 2001, pp. 957-960.

[21] A. Lohfink, P.-C. Eccardt, W. Benecke, and M. Meixner, "Derivation of a 1D cMUT model from FEM results for linear and nonlinear equivalent circuit simulation," in Proc. IEEE Ultrason. Symp., 2003, pp. 465-468.

[22] D. Pozar, Microwave Engineering. New York: Wiley, 1998.

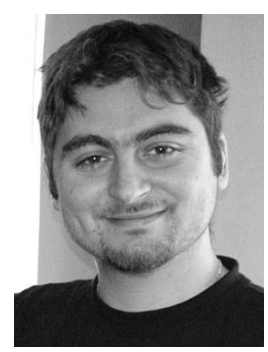

Selim Olcum was born in Chicago, IL, in 1981. He received his B.S. degree in electrical engineering from Bilkent University, Bilkent, Turkey, in 2003.

He worked as a guest researcher at the National Institute of Standards and Technology, Gaithersburg, MD, Semiconductor Division during the summers of 2002 and 2003 . He is currently working toward his Ph.D. degree in the Department of Electrical Engineering in Bilkent University where he has been a research assistant since 2003.

His current research interests include analysis, modeling and optimization of the cMUTs and generally microelectromechanical systems (MEMS).

He is a member of the Electron Device Society and UFFC Society since 2003 .

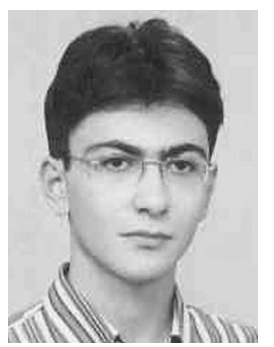

Muhammed N. Senlik was born in Isparta, Turkey, in 1981. He received his B.S. degree from Bilkent University, Bilkent, Turkey, in 2002 in electrical engineering. He is currently working toward his Ph.D. degree in the same department, where he has been a research assistant since 2002 .

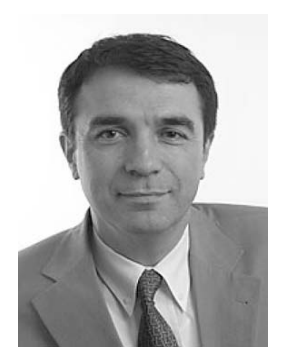

Abdullah Atalar was born in Gaziantep, Turkey, in 1954. He received a B.S. degree from Middle East Technical University, Ankara, Turkey, in 1974, M.S. and Ph.D. degrees from Stanford University, Stanford, CA, in 1976 and 1978, all in electrical engineering. From 1980 to 1986 he was an assistant professor in Middle East Technical University. In 1986, he joined Bilkent University, Bilkent, Turkey, as chairman of Electrical Engineering, where he is now a professor. Since 1996 he has been the Provost of the University.

He teaches courses on analog and digital integrated circuit design and microwave electronics. His current research interests include micromachined sensors and atomic force microscopy. He is a member of the Turkish Academy of Sciences and he serves on the Science Board of Tubitak. 